\title{
FRECUENCIA DE RABDOMIOSARCOMA CANINO EN EL LABORATO- RIO DE HISTOPATOLOGÍA VETERINARIA DE LA UNIVERSIDAD NACIONAL MAYOR DE SAN MARCOS (PERIODO 2000-2008)
}

\author{
Frequency of Canine Rhabdomyosarcoma in the Histopathological \\ Veterinary Laboratory of San Marcos University (Period 2000-2008)
}

Edwin Inga E. ${ }^{1}$, Nieves Sandoval C. ${ }^{1,2}$, Rosa Perales C. ${ }^{1}$, Alfonso Chavera C. ${ }^{1}$

\section{Resumen}

\begin{abstract}
El objetivo del presente estudio fue determinar la frecuencia del rabdomiosarcoma canino, según su clasificación histopatológica, diagnosticado en el laboratorio de histopatología de la Facultad de Medicina Veterinaria de la Universidad Nacional Mayor de San Marcos, Lima, Perú, en el periodo de enero de 2000 a diciembre de 2008. Fueron diagnosticados 63 casos de rabdomiosarcoma canino dentro de un total de 1125 casos de neoplasias caninas $(5.6 \pm 1.3 \%)$. De estos, sólo 47 casos pudieron ser clasificados, donde 27 casos $(57.4 \%)$ correspondieron a rabdomiosarcomas embrionarios, $19(40.4 \%)$ a rabdomiosarcomas alveolares y un caso $(2.1 \%)$ a rabdomiosarcoma pleomórfico. La región de mayor frecuencia de presentación para el tipo embrionario fue la cabeza y el cuello, siendo los canes de 6 a 10 años los más afectados.
\end{abstract}

Palabras clave: rabdomiosarcoma, clasificación histopatológica, caninos, neoplasia

\section{Abstract}

The purpose of this study was to determine the frequency of canine rhabdomyosarcoma based on its histopathological classification, that were diagnosed in the histopathology laboratory of the Veterinary Faculty of San Marcos University, Lima, Peru in the period of January 2000 to December 2008. A total of 63 cases of canine rhabdomyosarcoma were diagnosed out of 1125 canine neoplasic tumors $(5.6 \pm 1.3 \%)$. Among this, only 47 cases were possible to classify where 27 cases $(57.4 \%)$ corresponded to embryonal rhabdomyosarcoma, $19(40.4 \%)$ to alveolar rhabdomyosarcoma, and one case $(2.1 \%)$ to pleomorphic rhabdomyosarcoma. The most frequent region of the body affected by the embryonal type was the head and neck, where 6 to 10 year-old animals were the most affected.

Key words: rhabdomyosarcoma, histopathological classification, canine, neoplasia

\footnotetext{
${ }^{1}$ Laboratorio de Histología, Patología y Embriología Veterinaria, Facultad de Medicina Veterinaria, Universidad Nacional Mayor de San Marcos, Lima

${ }^{2}$ E-mail: nieves.sandovalchaupe@gmail.com
}

Recibido: 31 de mayo de 2012

Aceptado para publicación: 10 de marzo de 2013 


\section{INTRODUCCIÓN}

Los rabdomiosarcomas son tumores malignos originados a partir de los mioblastos o de células mesenquimales primitivas capaces de diferenciarse en células de músculo estriado (Cooper y Valentine, 2002). Si bien esta neoplasia se origina a partir del mesénquima embrionario que da origen al músculo esquelético estriado, también puede originarse en otras partes del cuerpo, incluyendo aquellas que carecen de músculo estriado como la vejiga urinaria, útero y cérvix (Malogalowkin et al., 1994; Cooper y Valentine, 2002; Breitfeld y Meyer, 2005).

Estos tumores se clasifican histológicamente como embrionarios, alveolares y pleomórficos. El rabdomiosarcoma botrioide es un subtipo de tumor embrionario y que tiene una apariencia macroscópica a manera de racimo de uva que protruye de membranas mucosas, tales como de la vejiga o del útero (Liptak y Forrest, 2007; Enziger y Weiss, 2008).

La presentación de rabdomiosarcomas en animales es muy diversa, siendo más común en el perro, seguido del caballo y el gato (Valentine y McGavin, 2007). Los animales afectados tienen generalmente menos de dos años de edad y los tumores se presentan usualmente en el cuello y la cavidad oral (especialmente la lengua), miocardio y tracto urinario (Cooper y Valentine, 2002; Valentine y McGavin, 2007).

El diagnóstico del rabdomiosarcoma es exclusivamente histopatológico; sin embargo, las actuales técnicas de histoquímica, microscopía electrónica y, sobre todo, el análisis inmunohistoquímico con anticuerpos monoclonales para la mioglobina y miosina, ayudan en el diagnóstico definitivo (Kobayashi et al., 2004). El esquema de clasificación histopatológica para el rabdomiosarcoma tiene significancia clínica para el pronóstico en humanos, pero esto no ha sido investigado en el canino. El potencial de metástasis y el pronóstico en perros con rabdomiosarcoma no ha sido determinado debido a que esta condición es raramente diagnosticada o tratada (Liptak y Forrest, 2007).

En el país no se han hecho intentos de clasificar histopatológicamente al rabdomiosarcoma canino de manera que se faciliten herramientas al clínico veterinario para la elaboración del tratamiento y formulación del pronóstico del animal afectado. El objetivo del estudio fue la clasificación histopalógica del rabdomiosarcoma canino a través de informes histopatológicos de nueve años de un laboratorio especializado de patología veterinaria en la ciudad de Lima, Perú.

\section{Materiales y Métodos}

El estudio se desarrolló en base a informes histopatológicos de canes en el Laboratorio de Histología, Patología y Embriología Veterinaria de la Facultad de Medicina Veterinaria, Universidad Nacional Mayor de San Marcos, Lima, Perú, cubriendo el periodo 1 de enero de 2000 al 31 de diciembre de 2008.

La clasificación histopatológica de los rabdomiosarcomas se hizo de acuerdo a los criterios de Cooper y Valentine (2002): embrionario, alveolar y pleomórfico (Cuadro 1). Se tomó en consideración las variables raza (pura y cruzada), edad (0-5, 6-10, 11-15 años), sexo, localización del tumor (cabeza y cuello, tracto urogenital, extremidades, otros), y la clasificación histopatológica. Se determinó la frecuencia del rabdomisarcoma canino con un intervalo de confianza del $95 \%$.

\section{Resultados}

En la evaluación de registros de nueve años se diagnosticaron 1125 neoplasias en caninos, 63 de los cuales correspondieron a rabdomiosarcomas $(5.6 \pm 1.3 \%)$. De estas, solo 47 pudieron ser clasificadas, donde el 
Cuadro 1. Clasificación y descripción histopatológica del rabdomiosarcoma canino ${ }^{1}$

\begin{tabular}{ll}
\hline $\begin{array}{l}\text { Tipo de } \\
\text { rabdomiosarcoma }\end{array}$ & Descripción histopatológica \\
\hline Embrionario & $\begin{array}{l}\text { Se observa diversos estadios del desarrollo embrionario del músculo } \\
\text { esquelético. Puede presentar zonas densas de celularidad densas con } \\
\text { otras mixoides. El estroma es escaso, con tendencia a la degeneración } \\
\text { mixoide con células hipercromáticas y citoplas ma eosinofílico. Las } \\
\text { células pueden ser bi- o multinucleadas. Puede verse rabdomioblastos }\end{array}$ \\
& y estrías cruzadas. \\
Alveolar & $\begin{array}{l}\text { Se observan agrupaciones de células redondas no diferenciadas } \\
\text { sosteniéndose sobre un soporte fibroso y a menudo a estructuras }\end{array}$ \\
& $\begin{array}{l}\text { similares a alveolos. Ante la pérdida de cohesión en los centros de los } \\
\text { nidos celulares, pueden verse células multinucleadas pero las estrías }\end{array}$ \\
& cruzadas son raras. \\
Pleomórfico & $\begin{array}{l}\text { Están constituidos por células grandes, pleomórficas en forma de } \\
\text { raquetas con núcleos muy bizarros y atípicos citoplasmáticos e } \\
\text { intensamente eosinofílicos. Puede haber fagocitosis por las células } \\
\text { tumorales y reacción inflamatoria moderadamente densa. }\end{array}$ \\
\hline
\end{tabular}

${ }^{1}$ Adaptado de Cooper y Valentine (2002)

$57.4 \%$ (27/47) de los casos de rabdomiosarcoma canino fue de tipo embrionario (Fig. 1), mientras que el $40.4 \%$ (19/47) fue de tipo alveolar (Fig. 2) y solo el $2.1 \%$ (1/47) fue de tipo pleomórfico (Fig. 3).

El $57.8 \%(26 / 45)$ de los casos se presentó en machos y el $42.2 \%$ (19/45) en hembras. Asimismo, la mayor frecuencia de casos ocurrió en canes menores de 10 años (40\% entre 0 a 5 años [18/45] y $44.4 \%$ entre 6 a 10 años [20/45]), siendo de $15.5 \%$ en canes de 11 a 15 años de edad (7/45).

Se encontró 10 casos en canes cruzados o de raza indeterminada (22.2\%), siendo la mayor frecuencia en canes de raza pura (77.8\%; 35/45). De estos, hubo 7 casos, tanto en las razas Bóxer y Rottweiler y uno o dos casos en cada una de las restantes 17 razas registradas en el estudio.

En relación a la localización del rabdomiosarcoma, las áreas más afectadas fueron la regiones de la cabeza y cuello con
$35.7 \%$ (15/42), las extremidades con $31.0 \%$ (13/42) y el tracto urogenital con $11.9 \%$ (5/ 42). Asimismo, otras áreas del cuerpo como la glándula mamaria y la región costal registraron el 21.4\% (9/42) de los casos. El tipo embrionario se presentó en el 50\% (12/24) de los casos en la región de cabeza y cuello, en tanto que el tipo alveolar se presentó en forma más uniforme en las diferentes regiones del cuerpo. En relación a la edad, el tipo embrionario se presentó mayormente en canes de 6 a 10 años de edad $(48.2 \%, 13 / 27)$ y el tipo alveolar en canes de 0 a 5 años de edad $(47.1 \%, 8 / 17)$.

\section{Discusión}

El 5.6\% de casos positivos a rabdomiosarcoma canino encontrado en los nueve años de registros histopatológicos fue bastante mayor a las observaciones de Silveira et al. (2007) quienes hallaron una frecuencia de rabdomiosarcoma del 3.2\% (2/63) dentro de 


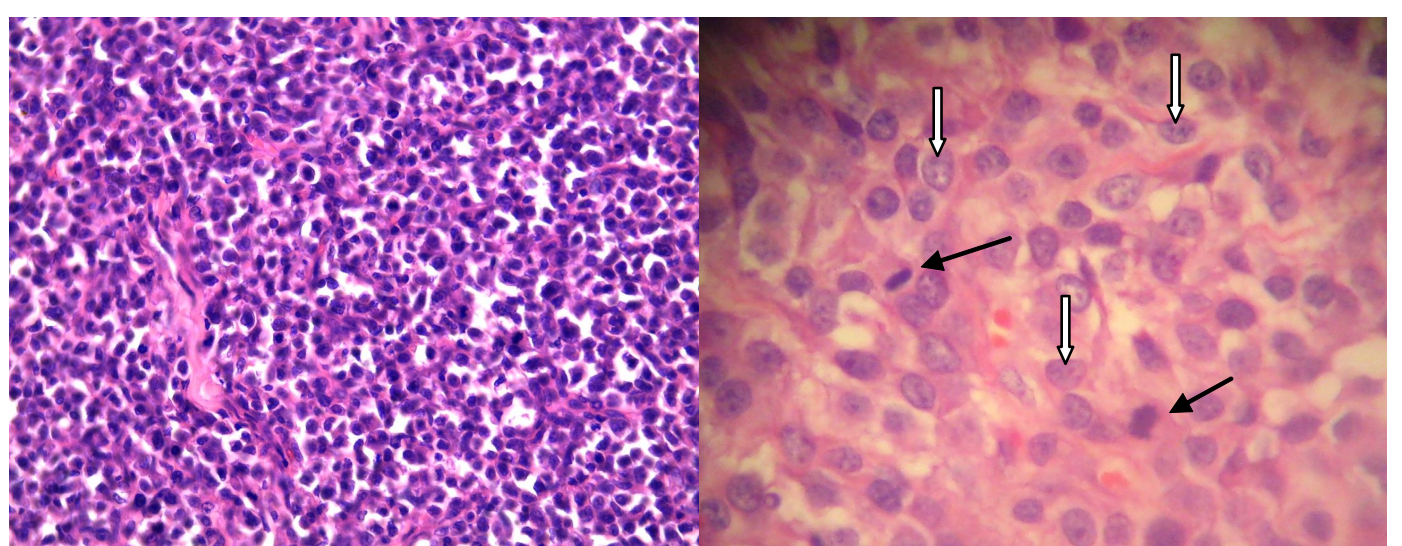

(A)

(B)

Figura 1. Imagen histopatológica de rabdomiosarcoma embrionario en un Rottweiler macho de 4 años con tumores en cabeza y cuello. (A). Se observan células redondas de diverso tamaño. H\&E, 100x. (B). Células redondas con citoplasma eosinófilo. Las flechas negras indican figuras de mitosis. 400x

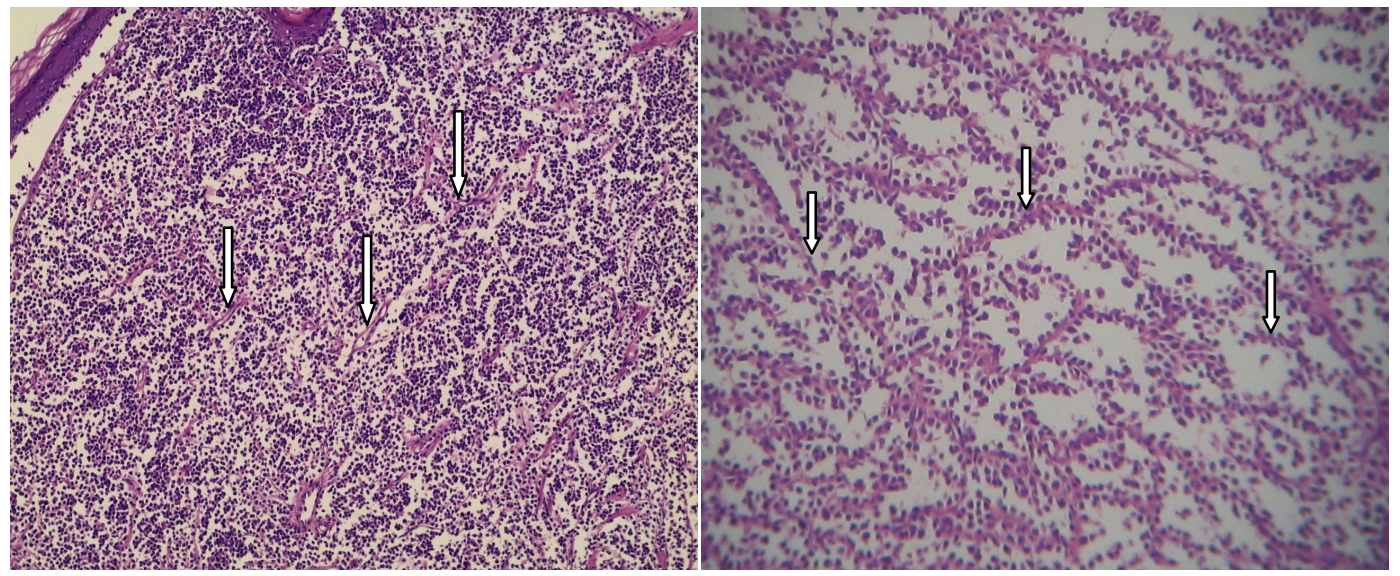

(A)

(B)

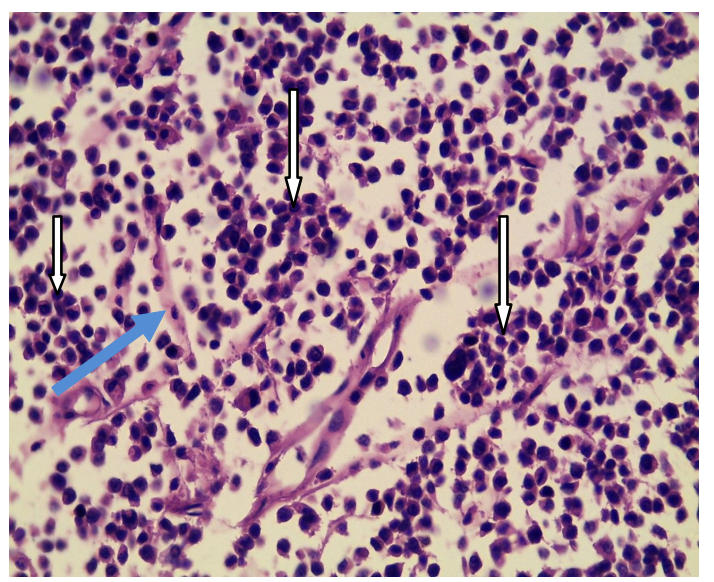

(C)

Figura 2. Imagen histopatológica de rabdomiosarcoma alveolar en un perro macho, cruzado, de 2.5 años, localizado en la región génitourinaria. (A) 50x. (B) 100x. Se observan células sostenidas en un conectivo fibroso que semejan alveolos. H\&E. (C) Células poligonales, mayormente con citoplasma eosinofílico, rodeadas por un delicado estroma «pseudo acinar» (flecha azul) 
las neoplasias mesenquimales. Asimismo, en un estudio retrospectivo de 20 años llevado a cabo en la Universidad de Cornell, EEUU, se reportó únicamente 12 casos de rabdomiosarcomas entre 83000 casos de neoplasias, lo cual representó el $0.00014 \%$ del total de neoplasias (Cooper y Valentine, 2002). Estas variaciones en las frecuencias pueden deberse a errores de diagnóstico, como refiere Cooper y Valentine (2002), que clasificaban a esta patología como neoplasia de células redondas o neoplasia benigna. Por otro lado, en todos los reportes, el tipo embrionario fue el de mayor predominancia.

Se evidenció una mayor frecuencia de casos en canes machos (57.8\%); sin embargo, no hay estudios que indiquen una predisposición del rabdomiosarcoma al sexo, con excepción del tipo botrioide de la vejiga urinaria en perros, el cual se presenta en una proporción de hembra/macho de 2:1 (Hulland, 1990; Takiguchi et al., 2002).

En el presente estudio, el grupo etario más afectado fue de 6 a 10 años (44.4\%). El rango de edad de perros afectados es amplio aunque con una media de 2 a 3 años de edad (Hulland, 1990; Kim et al., 1996; Yanoff et al., 1996; Kuwamura et al., 1998; Illanes, 2002; Takiguchi et al., 2002); habiendo un grupo importante de casos en canes mayores de seis años (Henderson et al., 1991; Block et al., 1995). En el humano, el rabdomiosarcoma es el más común de los tumores de los tejidos blandos en infantes y en individuos menores de 15 años de edad, comprendiendo casi el 50\% de las neoplasias de tejidos blandos en niños (MacArthur et al., 1992).

El tipo embrionario fue el más común dentro del grupo etario comprendido entre los 6 y 10 años, edad de madurez del perro. En comparación con el humano, este tipo de tumor se presenta generalmente en niños menores de 10 años y raramente en pacientes mayores de 40 años (Weiss y Goldblumb, 2009). Por otro lado, el tipo alveolar se presentó mayormente en canes menores de 5 años, en tanto que la mayor frecuencia de casos en el humano ocurre entre los 10 y 25 años (Moisés et al., 2007; Weiss y Goldblumb, 2009).

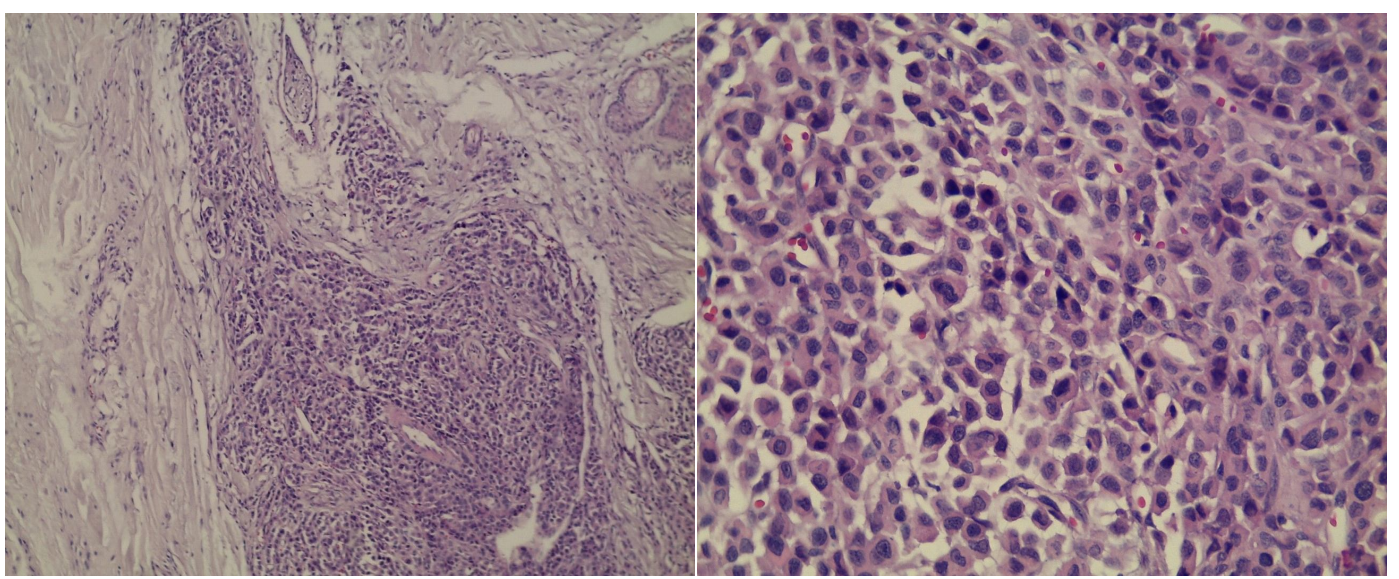

(A)

(B)

Figura 3. Imagen histopatológica de rabdomiosarcoma pleomórfico en una perra Pittbull hembra de 8 años de edad, localizado en la segunda y tercera falange. Se observa crecimiento desordenado de células muy grandes con citoplasma intensamente eosinofílico. H\&E. (A) 50x, (B) 100x 
La mayor frecuencia de casos se presentó en canes de las razas Rottweiler y Bóxer. No se ha podido establecer de manera clara un patrón de presentación del tipo de tumor de acuerdo a la raza, a excepción de la presentación del rabdomiosarcoma embrionario de característica macroscópica botrioide de la vejiga urinaria en perros (Hulland, 1990; Takiguchi et al., 2002), el cual presenta cierta predisposición en algunas razas grandes como el San Bernardo (Bae et al., 2007), Labrador Retriever (Kuwamura et al., 1998; Illanes, 2002; Ueno et al., 2002), Rottweiler (Kim et al., 1996), Pastor Alemán (Pérez et al., 1998) y Basset Hound (Kim et al., 1996).

La localización del tumor se presentó mayormente en la región de la cabeza y cue1lo, seguido de las extremidades, tracto urogenital y en otras regiones del cuerpo, coincidiendo con otros reportes en el perro (Cooper y Valentine, 2002; Nakaichi et al., 2007) y en el humano (Aguilar y Neciosup, 2006). El único caso de rabdomiosarcoma pleomórfico estuvo localizado en las extremidades.

\section{Conclusiones}

- Se encontró una frecuencia de $5.6 \pm$ $1.3 \%$ de casos de rabdomiosarcoma canino de un total de 1125 neoplasias diagnosticadas en un laboratorio de referencia histopatológica veterinaria en la ciudad de Lima, Perú.

- El tipo histopatológico de rabdomiosarcoma canino más frecuente fue el tipo embrionario (57.4\%).

- Se presentó mayormente en las razas puras (77.8\%), siendo las razas Bóxer y Rottweiler las más afectadas.

- La región más frecuente de presentación para el tipo embrionario fue la cabeza y el cuello.

\section{Literatura Citada}

1. Aguilar L, Neciosup S. 2006. Rabdomiosarcoma, estudio retrospectivo: Experiencia de una institución. Acta Cancerológica 34(1): 5-12.

2. Bae I, Kim Y, Pakhrin B, You M, Hwang C, Kim J, Kim D. 2007. Genitourinary rhabdomyosarcoma with systemic metastasis in a young dog. Vet Pathol 44: 518-520.

3. Block G, Clarke K, Salisbury S, Denicola D. 1995. Total laryngectomy and permanent tracheostomy for treatment of laryngeal rhabdomyosarcoma in a dog. J Am Anim Hosp Assoc 31:510-513.

4. Breitfeld P, Meyer W. 2005. Rhabdomyosarcoma. New windows of opportunity. Oncologist: 10(7): 518-527. [Internet] [1 abril 2011]. Disponible en: http://theoncologist.alphamedpress.org/ cgi/content/full/10/7/518

5. Cooper BJ, Valentine BA. 2002. Tumors of muscle. In: Tumors in domestic animals. $4^{\text {th }}$ ed. Iowa, USA: Iowa State Press. p 319-363.

6. Enzinger F, Weiss S. 2008. Rhabdomyosarcoma. In: Stamathis, G (ed). Soft tissue tumors. $2^{\text {nd }}$ ed. St. Louis, USA: CV Mosby. p 448-488.

7. Henderson RA, Powers RD, Perry L. 1991. Development of hypoparathyroidism after excision of laryngeal rhabdomyosarcoma in a dog. J Am Vet Med Assoc 198: 639-643.

8. Hulland T. 1990. Tumors of the muscle. In: Moulton JE (ed). Tumors in domestic animals. $3^{\text {rd }}$ ed. USA: University of California Press. p 93-100.

9. Illanes OG. 2002. Juvenile parameningeal rhabdomyosarcoma in a dog causing unilateral denervation atrophy of masticatory muscles. J Comp Pathol 126: 303-307. 
10. Kim, D, Hodgin E, Cho D, Varnado J. 1996. Juvenile rhabdomyosarcomas in two dogs. Vet Pathol 33: 447-450.

11. Kobayashi M, Sakai H, Hirata A. 2004. Expression of myogenic regulating factors, myogenin and myod, in two canine botryoid rhabdomyosarcomas. Vet Pathol 41: 275-277.

12. Kuwamura M, Yoshida H, Yamate J, Kotani T, Ohashi F, Sakuma S. 1998: Urinary bladder rhabdomyosarcoma (sarcoma botryoides) in a young Newfoundland dog. J Vet Med Sci 60: 619-621.

13. Liptak JM, Forrest LJ. 2007. Soft tissue sarcomas: overview and surgical management. In: Withrow SJ, Vail DV (eds). Small animal clinical oncology. $4^{\text {th }}$ ed. St Louis, USA: Elsevier. p 425-454.

14. MacArthur CJ, McGill TJ, Healy GB. 1992. Pediatric head and neck rhabdomyosarcoma. Clin Pediatr 31:66-70.

15. Malogalowkin MH, Rowland JM, Ortega JA. 1994. Rhabdomyosarcoma. In: Pochedly C (ed). Neoplastic diseases of childhood. Switzerland: Harwood Academic Publishers. p 779-814.

16. Moisés C, Oscanoa M, Hernández J, Gerónimo J, Gamero S, Chávez H. 2007. Rabdomiosarcoma alveolar de patrón sólido nasal, a propósito de un caso. Dermatol Pediatr Lat 5: 195-198.

17. Nakaichi M, Itamoto K, Hasegawa K. 2007. Maxillofacial rhabdomyosarcoma in the canine maxillofacial area. J Vet Med Sci 69: 65-67.
18. Pérez J, Perez-Rivero A, Montoya A, Martin A, Martin MP, Mozos E. 1998. Right-sided heart failure in a dog with primary cardiac rhabdomyosarcoma. $\mathbf{J}$ Am Anim Hosp Assoc 34: 206-211.

19. Silveira MF, Guim TN, Spader M, Schuch I, Gamba CO, Bonel-Raposo J, Fernandes CG. 2007. Estudo retrospectivo de 63 casos de sarcomas de tecido mole no período de 1980-2005. En: XIV Congreso de Iniciação Científica Estado do Rio Grande do Sul. Brasil.

20. Takiguchi M, Watanabe T, Okada H, Kudo T, Yamada K, Yasuda J, Hashimoto A 2002. Rhabdomyosarcoma (botryoid sarcoma) of the urinary bladderin a maltese. J Small Anim Pract 43: 269-271.

21. Ueno H, Kadosawa T, Isomura $H$, Okada Y, Ochiai K, Umemura T, et al. 2002: Perianal rhabdomyosarcoma in a dog. J Small Anim Pract 43: 217 220.

22. Valentine BA, McGavin MD. 2007. Skeletal muscle. In: McGavin M, Zachary JF (ed). Pathologic basis of veterinary disease. $4^{\text {th }}$ ed. St Louis: Mosby Elsevier. $1476 \mathrm{p}$.

23. Weiss S, Goldblum J. 2009. Tumores de partes blandas. $5^{\circ}$ ed. Barcelona: Elsevier. $1258 \mathrm{p}$.

24. Yanoff SR, Fuentealba C, Boothe $\mathrm{HW}$, Rogers KS. 1996. Tracheal defect and embryonal rhabdomyosarcoma in a young dog. Can Vet J 37: 172-173. 